\title{
REPORT OF THE IAU WORKING GROUP ON THE THEORY OF NUTATION
}

Dennis D. McCarthy

U. S. Naval Observatory

Washington, DC 20392 USA

ABSTRACT. Modern observations of celestial pole offsets indicate that small but significant corrections to the current IAU theories of nutation and precession can be detected. These corrections are of the order of a few milliseconds of arc. To meet the needs of those who require millisecond of arc accuracy in reference frame applications, the International Earth Rotation Service (IERS) publishes in weekly and monthly bulletins the most recent observations, along with predictions of these corrections. These appear to meet the needs of users. In view of the fact that the geophysical community has not adopted a model which describes the nutation and precession theory and that the current procedure of the IERS meets the need of high-accuracy users, there does not appear to be a need to identify a new IAU nutation theory at this time.

\section{Introduction}

The Working Group on Reference Systems (WGRS) was instituted at the IAU General Assembly in Baltimore in 1988. The Subgroup on Nutation was organized then to examine the various approaches which can be, and have been, taken towards the characterization and specification of this motion of the Earth. From this examination were to come recommendations regarding procedures to be used in describing this phenomenon. A new, analytical, closed theory of nutation was not necessarily the goal, although such an effort was not ruled out. The working group was charged with the responsibility of defining accessible, practical procedures regardless of the theoretical and observational foundations of the adopted approach to the problem in both its geophysical and astronomical aspects.

The International Earth Rotation Service (IERS) Standards (McCarthy 1989) recommends the use of the 1980 IAU Nutation Theory (Seidelmann 1982) based on the Wahr model (Wahr 1981). The IERS Central Bureau and the IERS Sub-bureau for Rapid Service and Predictions publish the observed corrections to the 1980 IAU nutation model and the predictions of these corrections. The predictions are based on a model utilizing derived corrections at nutation frequencies to extrapolate an improved nutation series.

The IAU 1980 Nutation Series is based on Kinoshita's (1977) rigid Earth theory, using Newcomb's Theory for the motion of the Earth, Brown's Theory for the motion of the Moon and the IAU 1976 system of Astronomical Constants. These theoretical coefficients are modified on the basis of Wahr's theory (1981) in the ratio of the amplitudes of each circular nutation relative to a realistic Earth model and to a rigid Earth model. This ratio is computed for an elliptical, rotating, elastic and oceanless 
Earth with a fluid, hydrostatically pre-stressed core. While this nutation series has proven adequate for many astronomical reductions, the introduction of high-precision VLBI and LLR observing techniques has revealed some significant inadequacies (Herring et al. 1986, Herring 1987, Zhu et a1. 1990, McCarthy and Luzum, 1990).

\section{Considerations}

Recently Kinoshita and Souchay (1990) have completed an improvement to Kinoshita's theory (1977) for a rigid Earth, using modern theories for the motion of the Earth and Moon, current values for the astronomical constants including the effect of the planetary perturbations. To enable the accurate reduction, on a common basis, of VLBI, LLR and SLR observations it would be desirable to arrive at an acceptable model of the Earth attempting to incorporate all known contributions at the one tenth milliarcsecond level. It should include the contribution of the nonhydrostatic core flattening as well as the oceanic and anelastic effects which have been shown to be significant and possible inner core effects. No geophysical model has become generally accepted although research in this area has been active (Molodenskiy and Kramer 1987, Mathews et al. 1989, Dehant 1990, Zhu et al. 1990,).

Until such a model is accepted it becomes necessary to provide standardized procedures to allow for systematic treatment of nutation for astronomy in general as well as the reduction of VLBI, LLR and SLR observations. It is the consensus of this Working Group that these corrections should be values based on geophysical models which produce corrections consistent with the observed results. This Working Group, then suggests the following recommendation.

\section{Recommendation}

The IAU Working Group on the Theory of Nutation

RECOGNIZING that a generally accepted non-rigid Earth theory of nutation including all known effects at the one tenth milliarc-second level is not yet available

\section{RECOMMENDS}

1. That those requiring accuracy of the nutation angles ( $\epsilon$ or $\psi \sin \epsilon_{0}$ ) numerically greater than $\pm 0: 002$ should continue to use the 1980 IAU Nutation Theory (Seidelmann 1982).

2. That those requiring nutation angle descriptions more accurate than $\pm 0: 002$ should make use of the Bulletins of the IERS which publish observations and predictions of the celestial pole offsets which are accurate to about $\pm 0: 0006$ for a period of up to six months.

3. That the IUGG be encouraged to develop and adopt an appropriate Earth model to serve as the basis for a new IAU Theory of Nutation. 


\section{Working Group Membership}

Members of the working group on the theory of nutation are:

Dennis D. McCarthy (chair)

U. S. Naval Observatory

Washington, DC 20392 USA

V. Dehant

Observatoire Royal de Belgique

3, Avenue Circulaire

B1180 Brussels, Belgium

Erwin Groten

Inst. of Physical Geodesy

Petersenstrasse 13

D-6100 Darmstadt, Germany

Thomas A. Herring

54-618 M.I.T.

77 Massachusetts Ave.

Cambridge, MA 02139 USA

G. H. Kaplan

U. S. Naval Observatory

Washington, DC 20392 USA

H. Kinoshita

Tokyo Astronomical Observatory

Mitaka Tokyo, 181 Japan
M. G. Rochester

Dept. of Earth Sciences

Memorial University of Newfoundland

St. John's, Newfoundland, Canada A1B $3 \times 7$

R. O. Vicente

Faculty of Sclences of Lisbon

R. Mestre Aviz, 30, R/C

1495 Lisboa, Portugal

$\mathrm{J}$. Vondrak

Astronomical Inst. of Czechoslovakia

Budecska, 6

12023 Praha 2, Czechoslovakia

J. M. Wahr

Department of Physics

Campus Box 390

University of Colorado

Boulder, CO 80309 USA

Ya. S. Yatskiv

Main Astronomical Observatory

Ukrainian Academy of Sclences

252127 Kiev USSR

\section{References}

Dehant, V.: 1990, Geophys. J. Int. 100, 477.

Herring, T.: 1987, In BIH Annual Report for 1987, pp. D-106-D108.

Herring, T. A., Gwinn, C. R., and Shapiro, I. I.: 1986, J. Geophys. Res. 91,4745 .

Kinoshita, H.: 1977, Celest. Mech. 15, 277.

Kinoshita, H. and Souchay, J.: 1990, Submitted to Celest. Mech.

McCarthy, D. D.: 1989, IERS Tech. Note 3, 14.

McCarthy, D. D. and Luzum, B. J.: 1990, submitted to Astron. J.

Mathews, P. M., Buffett, B. A., Herring, T. A., and Shapiro, I. I.: 1989,

Submitted to J. Geophys. Res.

Molodenskiy, S. M. and Kramer, M. V.: 1987, Izvest., Earth Phys. 23, 8. NEOS Annual Report for 1987

Sasao, T. and Wahr, J. M.: 1981, Geophys. J. R. Astron. Soc. 64, 729.

Seidelmann, P. K.: 1982, Celest. Mech. 27, 79.

Wahr, J. M.: 1981, Geophys. J. R. Astron. Soc. 64, 705. 
Woolard, E. W.: 1953, Astron. Pap. Amer. Eph. and Naut. Almanac 15, part 1.

Zhu, S. Y., and Groten, E.: 1989, Astron. J. 98, 1104.

Zhu, S. Y., Groten, E., and Reigber, C.: 1990, Astron. J. 99, 1024. 